\title{
Blood pressure reduction due to hemoglobin glycosylation in type 2 diabetic patients
}

\author{
Pedro Cabrales' \\ Miguel A Salazar Vázquez ${ }^{2,3}$ \\ Beatriz Y Salazar Vázquez $z^{3,4}$ \\ Martha Rodríguez-Morán ${ }^{5}$ \\ Marcos Intaglietta ${ }^{4}$ \\ Fernando Guerrero- \\ Romero ${ }^{5}$ \\ 'La Jolla Bioengineering Institute, \\ La Jolla, California, USA; ${ }^{2}$ Hospital \\ Regional No. I, of the Mexican \\ Social Security Institute, Victoria \\ de Durango, Dgo. Mexico; ${ }^{3}$ Faculty \\ of Medicine and Dept. of Physical \\ Chemistry, Universidad Juárez del \\ Estado de Durango, Victoria de \\ Durango, Dgo. Mexico; ${ }^{4}$ Department \\ of Bioengineering, University of \\ California, San Diego, La Jolla, \\ California, USA; ${ }^{5}$ Biomedical Research \\ Unit, of the Mexican Social Security \\ Institute, Victoria de Durango, Dgo. \\ Mexico
}

Objective: To test the hypothesis that glycosylation of hemoglobin constitutes a risk factor for hypertension.

Methods: A total of 129 relative uniform diabetic subjects ( 86 women and 42 men) were enrolled in a cross-sectional study. Exclusion criteria included alcohol consumption, smoking, ischemic heart disease, stroke, neoplasia, renal, hepatic, and chronic inflammatory disease. Systolic and diastolic pressures were recorded in subsequent days and mean arterial blood pressure (MAP) was determined. Hemoglobin glycosylation was measured by determining the percentage glycosylated hemoglobin $(\mathrm{HbA} 1 \mathrm{c})$ by means of the automated microparticle enzyme immunoassay test.

Results: MAP was found to be independent of the concentration of $\mathrm{HbA1c}$; however, correcting MAP for the variability in hematocrit, to evidence the level of vasoconstriction (or vasodilatation) showed that MAP is negatively correlated with the concentration of HbAlc ( $\mathrm{p}$ for trend $<0.05$ ), when patients treated for hypertension are excluded from the analysis. Patients treated for hypertension showed the opposite trend with increasing MAP as HbAlc increased ( $\mathrm{p}$ for the difference in trends $<0.05$ ).

Conclusions: Glycosylation per se appears to lead to blood pressure reduction in type 2 diabetic patients untreated for hypertension. Treatment for hypertension may be associated with a level of endothelial dysfunction that interferes with the antihypertensive effect of $\mathrm{HbAlc}$.

Keywords: diabetes, hemoglobin glycosylation, hypertension, hematocrit, nitric oxide

\section{Introduction}

Glycosylated hemoglobin ( $\mathrm{HbA} 1 \mathrm{c}, \% \mathrm{HbA} 1 \mathrm{c}$ relative to total hemoglobin, $\mathrm{Hb}$ ), indicating average plasma glucose over 3 months is highly correlated with most of the excess mortality risk of diabetes, which rises continuously beyond a $5 \%$ glycosylation index ( $\sim 0.7$ g/dl concentration) (van Hoeven and Factor 1990; Grossman and Messerli 1996; Khaw et al 2001). This relationship is attributed in part to an impairment of the rheological properties of blood, leading to increase red blood cell (RBC) stiffness, and alterations of plasma viscosity due to the interaction of uncontrolled plasma glucose levels with plasma proteins. There is also evidence that glycosylation of hemoglobin impairs nitric oxide (NO) related relaxation of human mesenteric vessels (Vallejo et al 2000) and it has been shown that $\mathrm{Hb}$ glycosylation alters $\mathrm{NO}$ binding to $\mathrm{Hb}$ thiols, lowering $\mathrm{NO}$ bioavailability and impairing vasodilatation in rabbit aortic rings (James et al 2004).

Microvascular NO bioavailability is a complex balance of NO production by the endothelium and NO scavenging and transport by $\mathrm{Hb}$ in RBCs. Blood flow and viscosity determine shear stress on the glycocalix/endothelium modulating the rate of production of NO by the endothelium via biochemical mechano transduction (Frangos et al 1996). This mechanism provides a form of autoregulation by which increases in peripheral vascular resistance due to anatomical or rheological changes increase shear stress and the release of endothelial dependant vasodilators, tending to normalize 
vascular resistance by lowering vessel tone. In normal healthy individuals additional autoregulatory mechanisms strive to optimize blood pressure and oxygen delivery, compensating for changes in blood viscosity due to the natural variability of this parameter due to fluid intake, alimentation, environmental adaptation, and some disease conditions. This effect was demonstrated by Martini and colleagues (2005) who increased hematocrit (Hct) and blood viscosity within the normal range of variability in healthy experimental animals, reducing peripheral vascular resistance and blood pressure and increasing cardiac index.

The $\% \mathrm{HbA} 1 \mathrm{c}$ increase in conditions of poor diabetic control is associated with increasing blood viscosity (Leiper et al 1984). Glycosylation of hemoglobin ( $\mathrm{Hb}$ ) may also change membrane lipid-protein interactions in RBCs changing their internal viscosity, altering viscoelastic properties of erythrocyte membranes and impairing RBC deformability (Watala et al 1992). RBC deformability in nephrotic patients is reduced, an effect associated with the abnormal carbohydrate metabolism level and the increased $\mathrm{HbA} 1 \mathrm{c}$ characteristic of this syndrome (Cecchin et al 1987).

Although glycosylation of $\mathrm{Hb}$ is a marker for significant cardiovascular risk, it is not clear whether it constitutes a risk. However, cumulative impairment of NO transport by HbAlc could constitute a significant risk factor in view of its direct implication in promoting hypertension, ischemia and enhanced production of oxygen free radicals. Notably these concepts arise primarily from in vitro tests. In the present study we investigated the relationship between $\mathrm{HbA} 1 \mathrm{c}$ index and concentration, blood glucose, mean arterial pressure (MAP), and Hct in a relatively uniform diabetic population of Victoria de Durango, a city in northern Mexico, to test the hypothesis that glycosylation of $\mathrm{Hb}$ constitutes a risk factor for hypertension.

\section{Material and methods}

A total of 129 subjects ( 86 women and 42 men) with previous diagnosis of type 2 diabetes were enrolled in a crosssectional study.

Protocol approval was obtained from the Mexican Social Security Institute (MSSI) Research Committee and informed consent was provided by each individual.

Exclusion criteria included alcohol consumption $\geq 30 \mathrm{~g}$ per week (Reynolds et al 2003), smoking, ischemic heart disease, stroke, neoplasia, renal, hepatic, and chronic inflammatory disease.

Blood pressure was measured on subsequent days, according with the VI Joint National Committee recommendation (JNC 1997). Systolic (SBP) and diastolic (DBP) blood pressures were recorded and MAP was determined using the relationship:

$$
\mathrm{MAP}=\mathrm{P}_{\text {diastolic }}+1 / 3\left(\mathrm{P}_{\text {systolic }}-\mathrm{P}_{\text {diastolic }}\right)
$$

Hemoglobin glycosylation was measured by determining the $\% \mathrm{HbA} 1 \mathrm{c}$ by means of the automated microparticle enzyme immunoassay (IMx, Abbot Laboratories, USA) test.

Mean arterial blood pressure was normalized vs. Hct using the relationship:

$$
M A P_{n i}=M A P_{i} \frac{H c t_{A v e}}{H c t_{i}}
$$

where $M A P_{n i}$ is the normalized blood pressure of an individual, $H c t_{\text {Ave }}$ is the average Hct in the population sample, and $H_{c t}$ is the Hct of the individual.

The $\% \mathrm{HbA} 1 \mathrm{c}$ was converted from a relative quantity to an absolute concentration in blood by multiplying this parameter by Hct, using the average Hct for this population of $43.0 \%$ and assuming that blood hemoglobin content is $14.5 \mathrm{~g} / \mathrm{dl}$. The following equation was used to normalize each individual:

$$
C H b A 1 c_{i}=\% H b A 1 c_{i} \times 14.5 \frac{H_{c t}}{H c t_{A v e}}
$$

where $C H b A 1 c_{i}$ is the concentration of HbA1c in blood in $\mathrm{g} / \mathrm{dl}$.

Blood samples $(3 \mathrm{ml})$ were drawn from the antecubital vein, 8 to $10 \mathrm{~h}$ after fasting, and collected in tubes with EDTA for Hct measurements. Hct was measured using a microhematocrit centrifuge $\left(13,000 \mathrm{~g}\right.$ for $3 \mathrm{~min}$ at $20^{\circ} \mathrm{C}$, Sol-Bat Centrifuge M-600, Readacrit L-10, Mexico). In addition, $3 \mathrm{ml}$ blood samples were drawn in tubes without anticoagulants for glucose measurements.

\section{Statistical analysis}

Pearson's analysis was performed to examine the correlation between $\% \mathrm{HbA1c}$, glucose level, blood pressure, and Hct. For the purpose of statistical analysis, all the skewed numerical data were transformed by $\log n$, to obtain a symmetrical distribution. Data were analyzed using the statistical package SPSS 12.0 (SPSS Inc., Chicago, IL, USA). A 95\% confidence interval $(95 \% \mathrm{CI})$ was considered, and $\mathrm{p}<0.05$ defined the level of statistical significance.

\section{Results}

Previously diagnosed diabetic patients that had undergone hypoglycemic treatment for a minimum period of 8 years were enrolled from the First Medical Care Level Offices of 
the MSSI in Victoria de Durango, Mexico. A total of 187 type 2 diabetic patients with average glycemia of $213 \pm 99 \mathrm{mg} / \mathrm{dl}$ were screened for hypertension and included in the study if SBP was $\leq 130 \mathrm{mmHg}$ and/or DBP $\leq 85 \mathrm{mmHg}$. Fifty-eight patients $(32 \%)$ were not included because they did not fulfill the inclusion-exclusion criteria; finally, a total of 129 diabetic patients were enrolled.

Amongst the included persons, 31 (24.0\%) were hypertensive and received pharmacological treatment with captopril $(n=22)$, nifedipine $(n=4)$, metoprolol $(n=3)$, and combined therapy $(n=2)$. None of the patients were treated with diuretics, and none of the treatment modalities is known to affect the concentration of HbAlc. Patients treated for hypertension were analyzed independently from the non treated diabetic patients; however the untreated population was not statistically different from the treated population with the exception of blood pressure $(p<0.001)$ as shown in Table 1.

The correlation between MAP and HbAlc \% for the whole population sample of 129 patients including patients treated and untreated for hypertension was negative but not statistically significant $(\mathrm{p}=0.24)$.

The correlation between MAP and $\mathrm{HbA} 1 \mathrm{c}$ when patients were separated into treated vs. non treated for hypertension was negative for untreated patients and positive for treated patients, however neither correlation was significant, although the difference in trends approached significance $(\mathrm{p}=0.06)$.

Normalizing MAP relative to Hct, yielded a negative nonsignificant $(\mathrm{p}=0.19)$ correlation between MAP of patients not treated for hypertension and $\% \mathrm{HbA} 1 \mathrm{c}$. Patients treated for hypertension showed a significant positive trend $(\mathrm{p}=0.02)$, and the difference in trends was highly significant $(\mathrm{p}=0.007)$, a result shown in Figure 1 .

Table I Population characteristics of diabetic type 2 patients treated for hypertension vs untreated

\begin{tabular}{lll}
\hline & Untreated & Treated \\
\hline Number of patients & 98 & 31 \\
Female/male & $67 / 31$ & $20 / I I$ \\
Age, years & $52.6 \pm 1.4$ & $51.5 \pm 4.2$ \\
Glucose & $220 \pm 78$ & $192 \pm 94$ \\
Cholesterol & $252 \pm 58$ & $240 \pm 52$ \\
Hematocrit, \% & $42.8 \pm 2.4$ & $43.7 \pm 4.5$ \\
HbAlc, \% & $9.3 \pm 2.6$ & $9.2 \pm 2.3$ \\
Mean arterial blood & $89.7 \pm 13.6$ & $101.8 \pm 12.5$ \\
pressure, mmHg & & \\
\hline
\end{tabular}

Notes: Mean \pm standard deviation. All parameters not statistically different $(p>0.05)$ with the exception of mean arterial blood pressure $(p<0.00 \mathrm{I})$.

Abbreviation: $\mathrm{HbAlc}$, glycosylated hemoglobin.

\section{Diabetic patients treated \& not treated for hypertension}

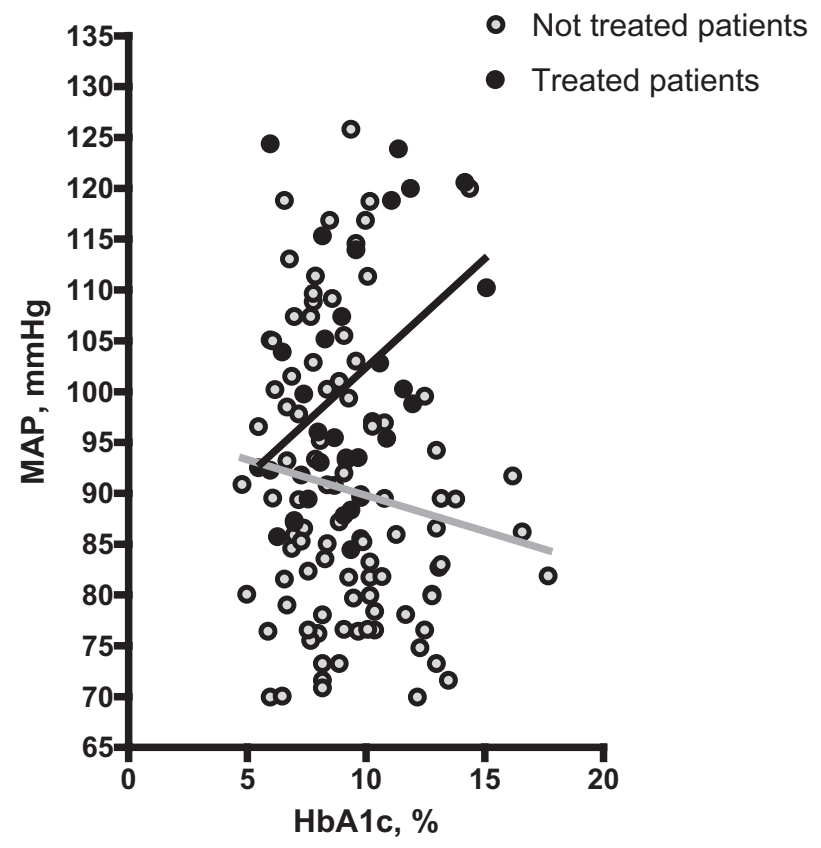

Figure I Mean arterial blood pressure (MAP, $\mathrm{mmHg}$ ) normalized relative to hematocrit for each patient versus glycosylation index. This normalization approximately excludes the effects of blood viscosity on MAP variability due to the distribution of Hct in the population. Patients untreated for hypertension had a negative MAP versus $\mathrm{Hb}$ glycosylation trend with slope: $-0.71 \pm 0.53 \mathrm{mmHg} / \% \mathrm{HbAlc}(\mathrm{p}<0.19)$. The trend for patients treated for hypertension was positive, $2.14 \pm 0.88(p<0.88)$. Therefore MAP is basically independent of \% $\mathrm{HbAl}$. Although neither trend was statically significant, the difference between trends was highly significant with $\mathrm{p}=0.007$.

Abbreviations: $\mathrm{Hb}$, hemoglobin; HbAlc, glycosylated hemoglobin; Hct, hematocrit.

The correlation between normalized MAP and $\mathrm{HbA1c}$ converted from $\%$ of blood hemoglobin to actual concentration $(\mathrm{g} / \mathrm{dl})$ is shown in Figure 2 for diabetics untreated and treated for hypertension. The trend had a statistically significant $(p=0.04)$ negative correlation for untreated patients and a positive nonsignificant $(\mathrm{p}=0.22)$ correlation for treated patients. The difference in trends however was statistically significant $(\mathrm{p}=0.04)$.

The decrease of blood pressure as a function of increased $\% \mathrm{HbA} 1 \mathrm{c}$ suggests that a factor affecting the relationship between MAP and Hct may be microalbuminuria, which is related to increased permeability caused by $\mathrm{HbAlc}$, and which could affect Hct. However, although there was a slightly positive functional dependence between these parameters, it was not statistically significant $\left(r^{2}=0.002\right.$ and $\left.p=0.66\right)$.

\section{Discussion}

The principal finding of this study is that MAP in a comparatively uniform population of diabetic of fairly similar racial 


\section{Diabetic patients treated \& not treated for hypertension}

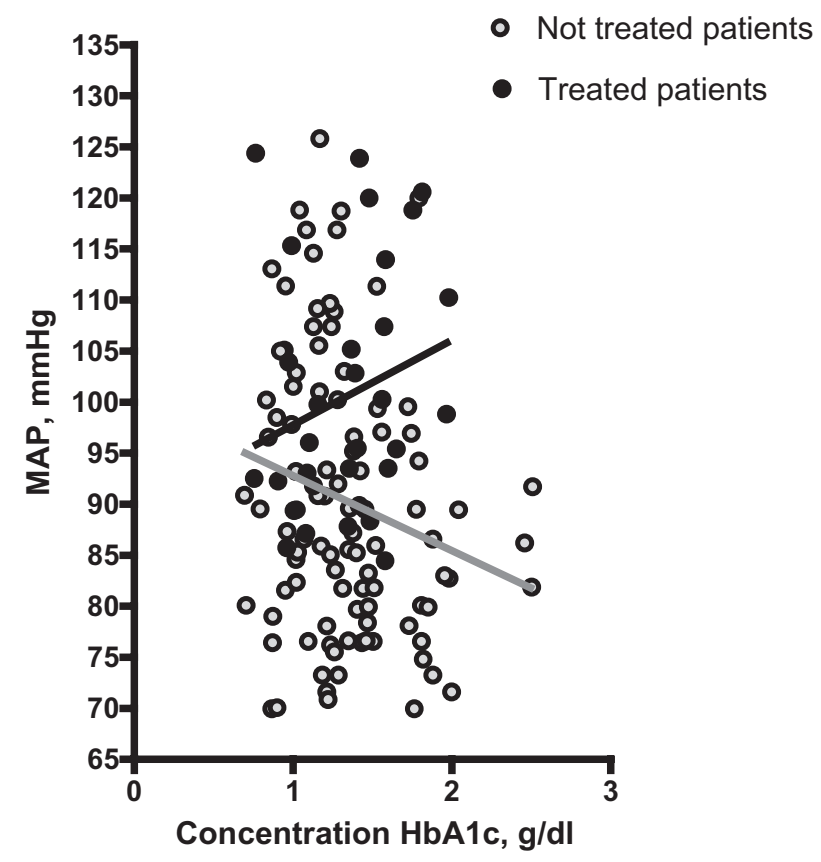

Figure 2 Normalized mean arterial blood pressure (MAP, $\mathrm{mmHg}$ ) as a function of concentration of $\mathrm{HbAlc}(\% \mathrm{HbAlc}$ times the calculated hemoglobin concentration in each patient). This normalization relates MAP directly to the concentration of glycosylated $\mathrm{Hb}$ in blood. Patients not treated for hypertension had a negative MAP vs. $\mathrm{HbAcl}$ concentration (slope $-7.36 \pm 3.57 \mathrm{mmHg} / \mathrm{HbAcl} \mathrm{g} / \mathrm{ml}$ ) that was significant $(p=0.04)$, a result opposite from that predicted by experimental studies in aortic rings. The trend for patient treated for hypertension was $8.31 \pm 0.67(p=0.22)$ which was not significant. The difference between slopes was significant with $\mathrm{p}=0.04$.

Abbreviations: $\mathrm{Hb}$, hemoglobin; HbAlc, glycosylated hemoglobin.

and ambient characteristic is unrelated to the glycosylation index of $\mathrm{Hb}$ when this ranges from 0.5 to $2.6 \mathrm{~g} / \mathrm{dl}$ plasma. Separating from this population individuals treated for hypertension shows that there is a statistically significant negative correlation between MAP normalized relative to Hct versus the concentration of $\mathrm{HbAlc}$.

Normalizing MAP relative to Hct renders the measured blood pressure independent of blood viscosity, and reveals whether MAP is a function of vascular regulation or the change in flow properties of blood. Thus normalizing MAP relative to Hct is a means for identifying effects on blood pressure due to vasoactivity vs. those due to the viscosity of blood determined by Hct, which presents a natural variability in the population. When the effect of blood viscosity is approximately neutralized by the normalization procedure blood pressure becomes directly related to the level of tone of the vasculature, ie, whether it is constricted or vasodilated, if we assume that anomalies of cardiac function, circulating blood volume, baroreceptor reflexes, etc., randomly affect blood pressure and are unrelated to $\mathrm{Hb}$ glycosylation. In the present case, it appears that individuals not treated for hypertension may be progressively vasodilated in proportion of the increase of total and/or relative $\mathrm{HbAc1}$ concentration increases. In this study we normalized blood pressure versus Hct since a previous study by Salazar-Vázquez and colleagues (2006), in the same population of diabetic patients, shows a "U" shaped distribution of MAP versus Hct. Regarding the statistical differences found between the different treatments of the data, it appears that the assessment of cardiovascular risk may be more evident when using hematocrit corrected MAP data versus \%HBA1c. However the fundamental difference between the two populations is expressed regardless of the difference in treatment of the data.

Since $8 \%-10 \% \mathrm{HbA} 1 \mathrm{c}$ is considered to be a threshold beyond which the effects of hemoglobin glycosylation become significant we determined MAP for patients not treated for hypertension below and above 9\% HbA1c, and found that below the threshold MAP was $86.2 \pm 3.9 \mathrm{mmHg}$ and $93.1 \pm 12.5 \mathrm{mmHg}$ above the threshold, the difference being statistically significant ( $p<0.001$ ), confirming the trend found when the data was analyzed as a whole. These results show that $\mathrm{Hb}$ glycosylation is not associated with the increase of the anatomical component of peripheral vascular resistance in individuals not treated for hypertension. Therefore the impairment of NO bioavalability leading to vasoconstriction postulated by in vitro studies in aortic rings does not appear to obtain in vivo (James et al 2004). It is furthermore apparent that solely on the basis of the effect that vasodilation and vasoconstriction has on blood pressure, there is no evidence that "...glycosylation impairs the NO vasodilator function of RBCs within the physiological range of tissue oxygenation" (James et al 2004). Remarkably James and colleagues (2004) found significant inhibition of hypoxic relaxation effects when $\mathrm{HbAlc}$ was $10.7 \%$ of human red blood cell was used in the perfusion of rabbit aortic rings. Conversely, our study failed to demonstrate an effect on blood pressure when individuals had HbAlc that was as much as $26 \%$ of the total RBCs. Thus the rabbit aortic rings study exposed to human glycosylated RBCs show vasoactivity that may be uncorrelated to that expected in a human cross-sectional study.

Translation and relevance of isolated tissue studies to in vivo conditions in humans is always a difficult endeavor, because the isolated tissue is not exposed to the full spectrum of phenomena operational in vivo. While it is undeniable that aortic rings exhibit a greater constriction tendency when 
exposed to glycosylated RBCs, our study shows that this phenomenon does not correspond to the increase of blood vessel tone dependant peripheral vascular resistance in the population at risk, and that the opposite may obtain.

There are several mechanisms that may in part explain our findings. A mechanism by which glycosylation of $\mathrm{Hb}$ is proposed to be vasoactive is via the formation of reactive oxygen species (ROS) (Angulo et al 1996) however this process is significantly mitigated inside RBCs. Glycosylation of $\mathrm{Hb}$ also lowers oxygen carrying capacity, promoting hypoxia and its related systemic vascular vasodilatory adaptations and responses (Paffett and Walker 2007).

Glycosylation of $\mathrm{Hb}$ as well as increased glucose levels tends to affect primarily RBC properties, lowering the RBC flexibility and increasing aggregability, the latter potentially increasing blood viscosity (Buhler et al 2001). Blood viscosity is one of the determinants of shear stress and peripheral vascular resistance via mechano-transduction. It should be noted that increased blood viscosity is not necessarily pathological, since it can be instrumental in increasing shear stress, enhancing the production of endothelial NO, compensating for the effect of viscosity on peripheral vascular resistance via vasodilatation. In this context decreased RBC flexibility has a moderate effect in increasing blood viscosity, which may in part explain the tendency for a negative correlation between glycosylation index and blood pressure.

The characteristic of the population studied were relatively uniform, in terms of age, duration of diabetes, cholesterol, and hematocrit, but differed significantly in MAP which was the cause for treatment of this segment of the population. The finding that the subgroup of patients treated for hypertension presents a statistically significant positive correlation with the increase of HbAlc suggests that in this population that requires a direct intervention for the control of MAP, endothelial dysfunction was significant. Consequently the presence of glycosylated $\mathrm{Hb}$ did not provide any compensatory effects at the level of the endothelium. In this context it appears that in diabetic patients that require treatment for hypertension hemoglobin glycosylation aggravates the consequences of endothelial dysfunction.

In conclusion, the existence of a negative, statistically significant correlation between MAP and the concentration of glycosylated $\mathrm{Hb}$ suggests that this parameter is not an indicator of hypertension. Increased HbAlc concentration should increase blood viscosity, peripheral vascular resistance, and blood pressure. Furthermore glycosylation of hemoglobin per se should impair the NO vasodilator function of RBC. However the lack or opposite consequence of these effects was found in a population of type 2 diabetic patients not treated for hypertension. The effects of hemoglobin glycosylation are mostly deduced from in vitro and tissue studies that do not correspond to physiological conditions, highlighting the difficulty of translating experimental findings to conditions in the human population. In vivo increased blood viscosity also leads to increased shear stress and therefore NO production by the endothelium, therefore if endothelial impairment is not present, then the potential, albeit small, increase in blood viscosity due to $\mathrm{Hb}$ glycosylation may be beneficial, although other rheological effects such as decreased RBC flexibility may adversely affect capillary perfusion. Finally diabetic patients requiring treatment for hypertension appear unable to compensate for the increased levels of $\mathrm{HbA} 1 \mathrm{c}$, confirming the presence of endothelial dysfunction, and possibly the impairment of hypoxic dilatation regulation.

\section{Acknowledgments}

This research was conducted with the financial support from the USPHS grants R01-HL62354 and R01-HL62318 to MI. Marcos Intaglietta is a member of the Board of Directors of the La Jolla Bioengineering Institute. In addition, this work was supported by grants from the Research Fund of Durango's State-National Science and Technology Council of Mexico (FOMIX Dgo-2002-C01-3760), the Research Promotion Fund of the Mexican Social Security Institute (FP 2002/366), and the Mexican Social Security Institute Foundation, Civil Association.

\section{References}

Angulo J, Sanchez-Ferrer CF, Peiro C, et al. 1996. Impairment of endothelium-dependent relaxation by increasing percentages of glycosylated human hemoglobin. Possible mechanisms involved. Hypertension, 28:583-92.

Buhler I, Walter R, Reinhart WH. 2001. Influence of D- and L-glucose on erythrocytes and blood viscosity. Eur J Clin Invest, 31:79-85.

Cecchin E, De Marchi S, Panarello G, et al. 1987. Rheological abnormalities of erythrocyte deformability and increased glycosylation of hemoglobin in the nephrotic syndrome. Am J Nephrol, 7:18-21.

Frangos JA, Huang TY, Clark CB. 1996. Steady shear and step changes in shear stimulate endothelium via independent mechanisms - superposition of transient and sustained nitric oxide production. Biochem Biophy Res Comm, 224:660-5.

Grossman E, Messerli FH. 1996. Diabetic and hypertensive heart disease. Ann Intern Med, 125:304-10.

James PE, Lang D, Tufnell-Barret T, et al. 2004. Vasorelaxation by red blood cells and impairment in diabetes: reduced nitric oxide and oxygen delivery by glycated hemoglobin. Circ Res, 94:976-83.

Khaw KT, Wareham N, Luben R, et al. 2001. Glycated haemoglobin, diabetes, and mortality in men in Norfolk cohort of European prospective investigation of cancer and nutrition (EPIC-Norfolk). BMJ, 322:15-18.

Leiper JM, Lowe GD, Anderson J, et al. 1984. Effects of diabetic control and biosynthetic human insulin on blood rheology in established diabetics. Diabetes Res, 1:27-30. 
Martini J, Carpentier B, Chavez Negrete A, et al. 2005. Paradoxical hypotension following increased hematocrit and blood viscosity. Am J Physiol Heart Circ Physiol, 289:H2136-43.

Paffett ML, Walker BR. 2007. Vascular adaptations to hypoxia: molecular and cellular mechanisms regulating vascular tone. Essays Biochem, 43:105-20.

Reynolds K, Lewis B, Nolen JD, et al. 2003. Alcohol consumption and risk of stroke: a meta-analysis. JAMA, 289:579-88.

Salazar-Vazquez BY, Intaglietta M, Rodriguez-Moran M, et al. 2006. Blood pressure and hematocrit in diabetes and the role of endothelial responses in the variability of blood viscosity. Diabetes Care, 29:1523-8.

[JNC] Joint National Committee on Prevention, Detection, Evaluation, and Treatment of High Blood Pressure. 1997. The Sixth Report of the Joint National Committee on Prevention, Detection, Evaluation, and Treatment of High Blood Pressure. Arch Intern Med, 157:2413-46.
Vallejo S, Angulo J, Peiro C, et al. 2000. Highly glycated oxyhaemoglobin impairs nitric oxide relaxations in human mesenteric microvessels. Diabetologia, 43:83-90.

van Hoeven KH, Factor SM. 1990. A comparison of the pathological spectrum of hypertensive, diabetic, and hypertensive-diabetic heart disease. Circulation, 82:848-55.

Watala C, Witas H, Olszowska L, et al. 1992. The association between erythrocyte internal viscosity, protein non-enzymatic glycosylation and erythrocyte membrane dynamic properties in juvenile diabetes mellitus. Int J Exp Pathol, 73:655-63. 